\title{
Bell inequality, separability and entanglement distillation
}

\author{
LI Ming ${ }^{1}$, FEI ShaoMing ${ }^{2,3 *} \&$ LI-JOST XianQing ${ }^{3,4}$ \\ ${ }^{1}$ College of Mathematics and Computational Science, China University of Petroleum, Dongying 257061, China; \\ ${ }^{2}$ School of Mathematical Sciences, Capital Normal University, Beijing 100048, China; \\ ${ }^{3}$ Max-Planck-Institute for Mathematics in the Sciences, Leipzig 04103, Germany; \\ ${ }^{4}$ Department of Mathematics, Hainan Normal University, Haikou 571158, China
}

Received August 3, 2010; accepted October 10, 2010

\begin{abstract}
In this review, we introduce well-known Bell inequalities, the relations between the Bell inequality and quantum separability, and the entanglement distillation of quantum states. It is shown that any pure entangled quantum state violates one of Bell-like inequalities. Moreover, quantum states that violate any one of these Bell-like inequalities are shown to be distillable. New Bell inequalities that detect more entangled mixed states are also introduced.
\end{abstract}

Bell inequality, entanglement, distillation

Citation: Li M, Fei S M, Li-Jost X Q. Bell inequality, separability and entanglement distillation. Chinese Sci Bull, 2011, 56: 945-954, doi: 10.1007/s11434-011-4395-1

The contradiction between local realism and quantum mechanics was first highlighted by the paradox of Einstein, Podolsky and Rosen (EPR) [1]. Nonlocality can be determined from violation of conditions, called Bell inequalities [2], that are satisfied by any local variable theory. In 1964, Bell formulated an inequality that is obeyed by any local hidden-variable theory. However, he showed that the EPR singlet state $\left|\psi_{+}\right\rangle=\frac{1}{\sqrt{2}}(|00\rangle+|11\rangle)$ violates the inequality. In fact, the Bell inequality provided the first possibility to distinguish experimentally between quantum-mechanical predictions and predictions of local realistic models. Bell inequalities are of great importance in understanding the conceptual foundations of quantum theory and investigating quantum entanglement, as they can be violated by quantum entangled states. On the other hand, violation of the inequalities is closely related to the extraordinary power of realizing certain tasks in quantum information processing, which outperforms its classical counterpart, such as building quantum protocols to decrease communication complexity [3] and providing secure quantum communication $[4,5]$.

One of the most important Bell inequalities is the ClauserHorne-Shimony-Holt (CHSH) inequality [6] for two-qubit

*Corresponding author (email: feishm@mail.cnu.edu.cn) systems. It can be generalized to the $N$-qubit case, known as the Mermin-Ardehali-Belinskii-Klyshko (MABK) inequality [7-9]. A set of multipartite Bell inequalities has been elegantly derived in terms of two dichotomic observables per site $[10,11]$. The set includes the MABK inequality as a special case [12] and can detect entangled states that the MABK inequality fails to detect. Ref. [13] introduced another family of Bell inequalities for $N$-qubit systems that are maximally violated by all Greenberger-Horne-Zeilinger states. A method of extending Bell inequalities from $n$ to $(n+1)$-partite states is described in [14]. In the higher dimensional bipartite case, Collins et al. [15] constructed a CHSH-type inequality for arbitrary $d$-dimensional (qudit) systems known as the Collins-Gisin-Linden-Masser-Popescu (CGLMP) inequality.

Gisin [16] presented a theorem in 1991 that states that any pure entangled two-qubit state violates the $\mathrm{CHSH}$ inequality. Specifically, the CHSH inequality is both sufficient and necessary for the separability of two-qubit states. Soon after, Gisin and Peres [17] provided an elegant proof of this theorem for the case of pure two-qudit systems. Chen et al. [18] showed that all pure entangled three-qubit states violate a Bell inequality. Nevertheless, it has remained an open problem for a long time whether Gisin's theorem can be generalized to the multi-qudit case. In addition, Bell inequalities that 
can detect more (mixed) entangled quantum states are being searched for.

Bell inequalities are also useful in verifying the security of quantum key distribution protocols $[19,20]$. There is a simple relation between nonlocality and distillability: if any two-qubit [21] or three-qubit [22] pure or mixed state violates a specific Bell inequality, then the state must be distillable. Dür showed that for the case $N \geqslant 8$, there exist $N$-qubit bound entangled (non-distillable) states that violate Bell inequalities [23]. However, Acín has demonstrated that for all states violating an inequality, there exists at least one kind of bipartite decomposition of the system such that a pure entangled state can be distilled [24, 25]. However generally it is an open problem whether violation of a Bell inequality implies distillability.

In this review, we first give a brief introduction of several important Bell inequalities in section 1. We introduce a set of Bell-like inequalities in section 2 that can be shown to be both sufficient and necessary for the separability of general pure bipartite quantum states in arbitrary dimensions. We then show that pure entangled states can be distilled from quantum mixed states that violate one of these Bell inequalities. New Bell operators are constructed in section 3 and used to detect more entangled quantum states. We further derive the maximal violation of such Bell inequalities. We give conclusions and remarks in section 4 .

\section{Some well-known Bell inequalities}

In this section we recall several useful Bell inequalities including the $\mathrm{CHSH}$ inequality, WWZB inequality (including the MABK inequality as a special case), CGLMP inequality and some other generalized inequalities.

\subsection{Bell inequalities for two and three-qubit systems}

The famous CHSH [6] inequality is a kind of improved Bell inequality that is more feasible for experimental verification. Suppose two observers, Alice and Bob, are separated spatially and share two qubits. Alice and Bob each measure a dichotomic observable with possible outcomes \pm 1 in one of two measurement settings: $A_{1}, A_{2}$ and $B_{1}, B_{2}$ respectively. The CHSH inequality is a constraint on correlations between Alice's and Bob's measurement outcomes if a local realistic description is assumed. The Bell function for the CHSH inequality has been given as [26]

$$
\mathcal{B}(\lambda)=A_{1}(\lambda)\left(B_{1}(\lambda)+B_{2}(\lambda)\right)+A_{2}(\lambda)\left(B_{1}(\lambda)-B_{2}(\lambda)\right),
$$

where $\lambda$ is a collection of local hidden variables and the variables $A_{i}(\lambda)$ and $B_{j}(\lambda)$ take values \pm 1 . According to the local hidden-variable theory, the statistical average of the Bell function must satisfy the inequality $[6,26],|\langle\mathcal{B}(\lambda)\rangle| \leqslant 2$, where the statistical average $\langle\mathcal{B}(\lambda)\rangle=\int \rho(\lambda) \mathcal{B}(\lambda) \mathrm{d} \lambda$ with $\rho(\lambda)$ the probability density distribution.
Quantum mechanically the statistical average of the Bell function is replaced by a quantum average of the corresponding operator given by

$$
\mathcal{B}=A_{1} \otimes B_{1}+A_{1} \otimes B_{2}+A_{2} \otimes B_{1}-A_{2} \otimes B_{2},
$$

where $A_{i}=\vec{a}_{i} \cdot \vec{\sigma}_{A}=a_{i}^{x} \sigma_{A}^{x}+a_{i}^{y} \sigma_{A}^{y}+a_{i}^{z} \sigma_{A}^{z}, B_{j}=\vec{b}_{j} \cdot \vec{\sigma}_{B}=$ $b_{j}^{x} \sigma_{B}^{x}+b_{j}^{y} \sigma_{B}^{y}+b_{j}^{z} \sigma_{B}^{z}, \vec{a}_{i}=\left(a_{i}^{x}, a_{i}^{y}, a_{i}^{z}\right)$ and $\vec{b}_{j}=\left(b_{j}^{x}, b_{j}^{y}, b_{j}^{z}\right)$ are real unit vectors satisfying $\left|\vec{a}_{i}\right|=\left|\vec{b}_{j}\right|=1$ with $i, j=1,2$, and $\sigma_{A, B}^{x, y, z}$ are Pauli matrices. The $\mathrm{CHSH}$ inequality says that if there exist local hidden-variable models to describe the system, the inequality

$$
|\langle\mathcal{B}\rangle| \leqslant 2
$$

must hold.

For entangled states, it is always possible to find suitable observables $A_{1}, A_{2}, B_{1}$ and $B_{2}$ such that inequality (3) is violated. For instance, taking $\left|\psi_{+}\right\rangle=(|01\rangle-|10\rangle) / \sqrt{2}, A_{1}=\sigma^{x}$, $A_{2}=\sigma^{z}, B_{1}=\left(\sigma^{x}+\sigma^{z}\right) / \sqrt{2}$, and $B_{2}=\left(\sigma^{x}-\sigma^{z}\right) / \sqrt{2}$, we obtain $|\langle\mathcal{B}\rangle|=2 \sqrt{2}$, which gives the maximal violation [27].

For three-qubit states, the Mermin inequality states that [7-9]

$$
\left|\left\langle A_{2} B_{1} C_{1}\right\rangle+\left\langle A_{1} B_{2} C_{1}\right\rangle+\left\langle A_{1} B_{1} C_{2}\right\rangle-\left\langle A_{2} B_{2} C_{2}\right\rangle\right| \leqslant 2,
$$

where observables $A_{i}, B_{i}$, and $C_{i}, i=1,2$, are associated with three qubits respectively. The maximal violation of the inequality (4) is 4 . The quantum mechanical violation of the Bell inequalities has been demonstrated experimentally, e.g. [28].

\subsection{Bell inequalities for multipartite qubit systems}

The MABK inequality is a kind of Bell inequality for multipartite qubits [7-9] whereas the WWZB inequality $[10,11]$ is a kind of generalization of the MABK inequality. Here we introduce the WWZB inequality and consider the MABK inequality as a special case of the WWZB inequality.

Consider an $\mathrm{N}$-qubit quantum system and allow each part to choose independently between two dichotomic observables $A_{j}, A_{j}^{\prime}$ for the $j$ th observer, specified by local parameters. Each measurement has two possible outcomes 1 and -1 . The WWZB quantum mechanical Bell operator is defined by

$$
\begin{aligned}
\mathcal{B}_{N}= & \frac{1}{2^{N}} \sum_{s_{1}, s_{2}, \cdots, s_{N}= \pm 1} S\left(s_{1}, s_{2}, \cdots, s_{N}\right) \\
& \times \sum_{k_{1}, k_{2}, \cdots, k_{N}= \pm 1} s_{1}^{k_{1}} s_{2}^{k_{2}} \cdots s_{N}^{k_{N}} \otimes_{j=1}^{N} O_{j}\left(k_{j}\right),
\end{aligned}
$$

where $S\left(s_{1}, s_{2}, \cdots, s_{N}\right)$ is an arbitrary function taking only values \pm 1 and $O_{j}(1)=A_{j}$ and $O_{j}(2)=A_{j}^{\prime}$ with $k_{j}=1,2$. It is shown in $[10,11]$ that local realism requires $\left|\left\langle\mathcal{B}_{N}\right\rangle\right| \leqslant 1$.

The MABK inequality is recovered by taking $S\left(s_{1}, s_{2}, \cdots\right.$, $\left.s_{N}\right)=\sqrt{2} \cos \left[\left(s_{1}+s_{2}+\cdots+s_{N}-N+1\right) /(\pi / 4)\right]$ in $(5)$.

Employing an inductive method from the $(N-1)$-partite WWZB Bell inequality to the $N$-partite inequality, a family 
of Bell inequalities was presented in [13]. The new Bell operator is defined by

$$
\mathcal{B}_{N}=\mathcal{B}_{N-1} \otimes \frac{1}{2}\left(A_{N}+A_{N}^{\prime}\right)+I_{N-1} \otimes \frac{1}{2}\left(A_{N}-A_{N}^{\prime}\right),
$$

where $\mathcal{B}_{N-1}$ represents the normal WWZB Bell operators defined in (5). Such new Bell operators yield violation of the Bell inequality for the generalized GHZ states, $|\psi\rangle=$ $\cos \alpha|00 \cdots 0\rangle+\sin \alpha|11 \cdots 1\rangle$, in the whole parameter region of $\alpha$ and for any number of qubits, thus overcoming the drawback of the WWZB inequality. In the three-qubit case, one can construct three different Bell operators from $\mathcal{B}_{2}$ by taking the approach of (6). The corresponding three Bell inequalities can distinguish full separability, detailed partial separability and true entanglement [29].

\subsection{Bell inequalities for high-dimensional systems}

For bipartite high-dimensional quantum systems, we introduce the CGLMP inequality given in [15]. We consider the standard Bell-type experiment: two spatially separated observers, Alice and Bob, share a copy of a pure two-qudit state $|\psi\rangle \in \mathbb{C}^{d} \otimes \mathbb{C}^{d}$ in the composite system. Suppose that Alice and Bob both have the choice of performing one of two different projective measurements, each of which has $d$ possible outcomes. Let $A_{1}$ and $A_{2}$ denote observables measured by Alice and $B_{1}$ and $B_{2}$ the observables measured by Bob. Each measurement has $d$ possible outcomes: $0,1, \cdots, d-1$. Any local variable theory must then obey the well-known CGLMP inequality [15]:

$$
\begin{aligned}
I_{d} \equiv & \sum_{k=0}^{\left[\frac{d}{2}\right]-1}\left(1-\frac{2 k}{d-1}\right)\left\{\left[P\left(A_{1}=B_{1}+k\right)\right.\right. \\
& +P\left(B_{1}=A_{2}+k+1\right)+P\left(A_{2}=B_{2}+k\right) \\
& \left.+P\left(B_{2}=A_{1}+k\right)\right]-\left[P\left(A_{1}=B_{1}-k-1\right)\right. \\
& +P\left(B_{1}=A_{2}-k\right)+P\left(A_{2}=B_{2}-k-1\right) \\
& \left.\left.+P\left(B_{2}=A_{1}-k-1\right)\right]\right\} \leqslant 2 .
\end{aligned}
$$

Here $[x]$ denotes the integer part of $x$. The joint probability $P\left(A_{a}=B_{b}+m\right)=\sum_{j=0}^{d-1} P\left(A_{a}=j, B_{b}=j-m\right), a, b=1,2$, in which the measurements $A_{a}$ and $B_{b}$ have outcomes that differ by $m(\bmod d)$.

Chen et al. show that all bipartite entangled states violate the CGLMP inequality [30], which gives a detailed proof of Gisin's Theorem for two-qudit quantum systems.

Let $X_{j}^{[1]}$ and $X_{j}^{[2]}$, where $j=1,2$, denotes the two observables for the $j$ th party. Each has $d$ possible outcomes: $x_{j}^{[1]}, x_{j}^{[2]}=0,1, \cdots, d-1$. Fu introduced the correlation function $Q_{i j}[31]$,

$$
Q_{i j}=\frac{1}{S} \sum_{m, n=0}^{d-1} f^{i j}(m, n) P\left(X_{1}^{[i]}=m, X_{2}^{[j]}=n\right),
$$

where $S=(d-1) / 2$ is the spin of the particle for the $d$ dimensional system, and $P\left(X_{1}^{[i]}=m\right.$ and $\left.X_{2}^{[j]}=n\right)$ are the joint probabilities. $f^{i j}(m, n)=S-M[\varepsilon(i-j)(m+n), d]$; $\varepsilon(x)=1$ for $x \geqslant 0$ and -1 for $x<0 ; M(x, d)=x \bmod d$, $0 \leqslant M(x, d) \leqslant d-1$. On the basis of these correlation functions, a tight Bell inequality for two-qudit systems is obtained:

$$
I_{d}^{[2]} \equiv Q_{11}+Q_{12}-Q_{21}+Q_{22} \leqslant 2 .
$$

Inequality (9) is equivalent to the CGLMP inequality.

Chen et al. further generalized this kind of correlation function to arbitrary $N$-qudit systems [32]. Let $X_{j}^{[1]}$ and $X_{j}^{[2]}$, where $j=1,2, \cdots, N$, denote the two observables for the $j$ th party. Each has $d$ possible outcomes: $x_{j}^{[1]}, x_{j}^{[2]}=0,1, \cdots, d-$ 1. The generalized correlation functions are then defined as

$$
\begin{aligned}
Q_{i_{1}, \cdots, i_{N}}= & \frac{1}{S} \sum_{x_{1}^{\left[i_{1}\right]}=0}^{d-1} \cdots \sum_{x_{N}^{\left[i_{N}\right]}=0}^{d-1} f^{i_{1} \cdots i_{N}}\left(x_{1}^{\left[i_{1}\right]}, \cdots, x_{N}^{\left[i_{N}\right]}\right) \\
& \times P\left(X_{1}^{\left[i_{1}\right]}=x_{1}^{\left[i_{1}\right]}, \cdots, X_{N}^{\left[i_{N}\right]}=x_{N}^{\left[i_{N}\right]}\right),
\end{aligned}
$$

where $S=\frac{d-1}{2}, f^{i_{1} \cdots i_{N}}\left(x_{1}^{\left[i_{1}\right]}, \cdots, x_{N}^{\left[i_{N}\right]}\right)=S-M\left[(-1)^{\chi}\right.$ $\left.\times\left(\sum_{j=1}^{N} x_{j}^{\left[i_{j}\right]}\right), d\right]$ and $\chi=\Pi_{j=1}^{N} i_{j}$. According to these correlation functions, the generalized multipartite Bell inequality can be written as

$$
\begin{gathered}
I_{d}^{[2 N]}=Q_{1 \cdots 1}+Q_{1212 \cdots 12}+Q_{2121 \cdots 21}-Q_{2 \cdots 2} \leqslant 2, \\
I_{d}^{[2 N+1]}=Q_{1 \cdots 1}+Q_{1212 \cdots 21}+Q_{2121 \cdots 12}-Q_{2 \cdots 2} \leqslant 2 .
\end{gathered}
$$

\subsection{Bell inequalities for many-setting systems}

Gisin [33] investigated the CHSH inequalities for two-qubit quantum systems with many settings. Let $a_{j}= \pm 1$ and $b_{j}= \pm 1$ for all indices $j=1,2, \cdots, n$. The inequality

$$
\sum_{j=1}^{n}\left(\sum_{k=1}^{n+1-j} a_{j} b_{k}-\sum_{k=n+2-j}^{n} a_{j} b_{k}\right) \leqslant\left[\frac{n^{2}+1}{2}\right]
$$

can be easily derived, where $[x]$ denotes the largest integer smaller or equal to $x$. Inspired by this, one can set the Bell operator to be

$$
\mathcal{B}=\sum_{j=1}^{n}\left(\sum_{k=1}^{n+1-j} A_{j} B_{k}-\sum_{k=n+2-j}^{n} A_{j} B_{k}\right),
$$

where $A_{j}=\vec{a}_{j} \cdot \vec{\sigma}, B_{k}=\vec{b}_{k} \cdot \vec{\sigma}, \vec{a}=\left(a_{x}, a_{y}, a_{z}\right)$ and $\vec{b}=$ $\left(b_{x}, b_{y}, b_{z}\right)$ are real unit vectors, and $\vec{\sigma}=\left(\sigma^{x}, \sigma^{y}, \sigma^{z}\right)$. The Bell inequalities for two-qubit systems with many settings are then

$$
\langle\mathcal{B}\rangle \leqslant\left[\frac{n^{2}+1}{2}\right] \text {. }
$$

The ratio of the maximal violation of the inequality decreases with the increasing number of settings. The usual two-setting CHSH inequality has a maximal violation ratio $\sqrt{2}$. For large $n$-settings, the ratio tends to $4 / \pi \sim 1.273$ [33].

The authors studied $d \otimes d$-dimensional bipartite systems with $d$ a prime integer [34]. Two observers are allowed each 
to choose one of $d$ variables. Consider a classical Bell function,

$$
\mathcal{B}(\lambda)=\frac{1}{d-1} \sum_{n, i, j=1}^{d-1} \omega^{n i j} A_{i}^{n}(\lambda) B_{j}^{n}(\lambda),
$$

where $\omega=\mathrm{e}^{\frac{i 2 \pi}{d}}$ is the primitive $d$-th root of unity, $A_{i}(\lambda)=$ $\omega^{a_{i}(\lambda)}$ and $B_{j}(\lambda)=\omega^{b_{j}(\lambda)}$ with $a_{i}(\lambda)$ and $b_{j}(\lambda)$ integer-valued functions of hidden variable $\lambda$.

The quantum Bell operator, corresponding to the classical Bell function, is given by

$$
\mathcal{B}=\frac{1}{d-1} \sum_{n, i, j=1}^{d-1} \omega^{n i j} A_{i}^{n} B_{j}^{n},
$$

where $A_{i}$ and $B_{j}$ are local unitary operators with eigenvalues $1, \omega, \omega^{2}, \cdots, \omega^{d-1}$. It is shown that the statistical average of the Bell operator satisfies

$$
-\frac{d^{2}}{d-1} \leqslant\langle\mathcal{B}\rangle \leqslant \frac{d(2 d-3)}{d-1} .
$$

This Bell inequality is maximally violated quantum mechanically by mutually unbiased measurements of a maximally entangled state, whereas other Bell inequalities for high-dimensional systems such as the CGLMP inequality [15] and that of Son et al. [35] do not have such a property.

\section{Gisin's theorem}

In this section we introduce a set of Bell-like inequalities that can be shown to be both sufficient and necessary for separability of general pure bipartite quantum states in arbitrary dimensions [36].

\subsection{Bell inequalities for bipartite quantum systems}

We first consider general $N \times M$ bipartite quantum systems in vector space $\mathcal{H}_{\mathcal{A B}}=\mathcal{H}_{\mathcal{A}} \otimes \mathcal{H}_{\mathcal{B}}$ with dimensions $\operatorname{dim} \mathcal{H}_{\mathcal{A}}=M$ and $\operatorname{dim} \mathcal{H}_{\mathcal{B}}=N$. We aim to find Bell inequalities like (3) such that any quantum entangled states violates a Bell inequality.

Let $L_{\alpha}^{A}$ and $L_{\beta}^{B}$ be the generators of special unitary groups $S O(M)$ and $S O(N)$ respectively. The $M(M-1) / 2$ generators $L_{\alpha}^{A}$ are given by $\{|j\rangle\langle k|-| k\rangle\langle j|\}, 1 \leqslant j<k \leqslant M$, where $|i\rangle$, $i=1, \cdots, M$, is the usual orthonormal basis of $\mathcal{H}_{\mathcal{H}}$, a column vector with the $i$ th row 1 and the rest zeros. $L_{\beta}^{B}$ is similarly defined. The matrix operators $L_{\alpha}$ (resp. $L_{\beta}$ ) have $M-2$ (resp. $N-2$ ) rows and $M-2$ (resp. $N-2$ ) columns that are identically zero. We define the operators $A_{i}^{\alpha}$ (resp. $B_{j}^{\beta}$ ) from $L_{\alpha}$ (resp. $L_{\beta}$ ) by replacing the four entries in the positions of the two nonzero rows and two nonzero columns of $L_{\alpha}$ (resp. $L_{\beta}$ ) with the corresponding four entries of the matrix $\overrightarrow{a_{i}} \cdot \vec{\sigma}$ (resp. $\overrightarrow{b_{j}} \cdot \vec{\sigma}$ ), and keeping the other entries of $A_{i}^{\alpha}$ (resp. $B_{j}^{\beta}$ ) zero. We define the Bell operators as

$$
\mathcal{B}_{\alpha \beta}=\tilde{A}_{1}^{\alpha} \otimes \tilde{B}_{1}^{\beta}+\tilde{A}_{1}^{\alpha} \otimes \tilde{B}_{2}^{\beta}+\tilde{A}_{2}^{\alpha} \otimes \tilde{B}_{1}^{\beta}-\tilde{A}_{2}^{\alpha} \otimes \tilde{B}_{2}^{\beta},
$$

where $\tilde{A}_{i}^{\alpha}=L_{\alpha} A_{i}^{\alpha} L_{\alpha}^{\dagger}, \tilde{B}_{j}^{\beta}=L_{\beta} B_{j}^{\beta} L_{\beta}^{\dagger}$, and $i, j=1,2$.

Theorem 1 Any bipartite pure quantum state is entangled if and only if at least one of the following Bell inequalities is violated [36]:

$$
\left|\left\langle\mathcal{B}_{\alpha \beta}\right\rangle\right| \leqslant 2,
$$

where $\alpha=1,2, \cdots, \frac{M(M-1)}{2}, \beta=1,2, \cdots, \frac{N(N-1)}{2}$.

Proof Assume that the state $|\psi\rangle$ violates one of the Bell inequalities in (19): i.e. there exist $\alpha_{0}$ and $\beta_{0}$ such that $\left|\left\langle\mathcal{B}_{\alpha_{0} \beta_{0}}\right\rangle\right|>2$. Equivalently one has that the state $|\psi\rangle_{\alpha_{0} \beta_{0}}=$ $\frac{L_{\alpha_{0}}^{A} \otimes L_{\beta_{0}}^{B}|\psi\rangle}{\| L_{\alpha_{0}}^{A} \otimes L_{\beta_{0}}^{B}|\psi\rangle \|}$ violates the CHSH inequality in (3). As the local operation $L_{\alpha_{0}}^{A} \otimes L_{\beta_{0}}^{B}$ does not change the separability of a state, $|\psi\rangle$ must be entangled.

Now assume that $|\psi\rangle \in \mathcal{H}_{\mathcal{A B}}$ is an entangled state. We prove that at least one of the Bell inequalities in (19) is violated. Set $\rho=|\psi\rangle\langle\psi|$. By projecting $|\psi\rangle$ onto $2 \times 2$ subsystems [37], we get the following pure states

$$
\rho_{\alpha \beta}=\frac{L_{\alpha}^{A} \otimes L_{\beta}^{B} \rho\left(L_{\alpha}^{A}\right)^{\dagger} \otimes\left(L_{\beta}^{B}\right)^{\dagger}}{\left\|L_{\alpha}^{A} \otimes L_{\beta}^{B} \rho\left(L_{\alpha}^{A}\right)^{\dagger} \otimes\left(L_{\beta}^{B}\right)^{\dagger}\right\|},
$$

where $\alpha=1,2, \cdots, \frac{M(M-1)}{2}, \beta=1,2, \cdots, \frac{N(N-1)}{2}$, and $\|X\|=$ $\sqrt{\operatorname{Tr}\left(X X^{\dagger}\right)}$. Here $\rho_{\alpha \beta}$ are pure states with rank one. As the matrix $L_{\alpha}^{A} \otimes L_{\beta}^{B}$ has $M N-4$ rows and $M N-4$ columns that are identically zero, there are at most $4 \times 4=16$ nonzero elements in the matrix $\rho_{\alpha \beta}$. The states $\rho_{\alpha \beta}$ are called "two-qubit" states in this sense.

The concurrence of $|\psi\rangle$ is defined by $C(|\psi\rangle)=$ $\sqrt{2\left(1-\operatorname{Tr}\left(\rho_{A}^{2}\right)\right)}$ with $\rho_{A}=\operatorname{Tr}_{B}(\rho)$ the reduced density matrix of $\rho$ by tracing over the subsystem $B[39,40]$. A pure quantum state $|\psi\rangle$ can be generally expressed as $|\psi\rangle=$ $\sum_{i=1}^{M} \sum_{j=1}^{N} a_{i j}|i j\rangle, a_{i j} \in \mathbb{C}$, in the computational basis $|i\rangle$ and $|j\rangle$ of $\mathcal{H}_{\mathcal{A}}$ and $\mathcal{H}_{\mathcal{B}}$ respectively, where $i=1, \cdots, M$ and $j=1, \cdots, N$. Therefore, the concurrence can be expressed as

$$
C(|\psi\rangle)=\sqrt{\sum_{\alpha=1}^{M} \sum_{\beta=1}^{N}\left|C\left(\rho_{\alpha \beta}\right)\right|^{2}},
$$

where $\rho_{\alpha \beta}$ are defined in (20). Since we have assumed that $|\psi\rangle$ is an entangled quantum state, $C(|\psi\rangle)$ must be nonzero: i.e. at least one of $\rho_{\alpha \beta}$, say $\rho_{\alpha_{0} \beta_{0}}$, has nonzero concurrence: $C\left(\rho_{\alpha_{0} \beta_{0}}\right)>0$. As discussed above, $\rho_{\alpha_{0} \beta_{0}}$ is actually a "twoqubit" quantum pure state. It has been shown that an entangled two-qubit pure state must violate the Bell inequality (3) $[16,17]$. Therefore, the inequality $\left|\left\langle\mathcal{B}_{\alpha_{0} \beta_{0}}\right\rangle\right| \leqslant 2$ is violated.

As an example we consider a bipartite $3 \times 3$ quantum state $|\psi\rangle$ with Schmidt decomposition $|\psi\rangle=\sum_{i=1}^{3} \lambda_{i}\left|i_{A}\right\rangle\left|i_{B}\right\rangle, \lambda_{i} \geqslant 0$ and $\sum_{i} \lambda_{i}^{2}=1$. The concurrence of $|\psi\rangle$ is given by

$$
C(|\psi\rangle)=4\left(\lambda_{1} \lambda_{2}\right)^{2}+4\left(\lambda_{1} \lambda_{3}\right)^{2}+4\left(\lambda_{2} \lambda_{3}\right)^{2} .
$$


If $|\psi\rangle$ is entangled, the concurrence must have at least one nonzero term in (22), say $\lambda_{1} \lambda_{3} \neq 0$. The corresponding observables are $L_{2}^{A}=L_{2}^{B}=\left(\begin{array}{ccc}0 & 0 & 1 \\ 0 & 0 & 0 \\ -1 & 0 & 0\end{array}\right)$ and

$$
\rho=\left(\begin{array}{ccccccccc}
\lambda_{3}^{2} & 0 & 0 & 0 & 0 & 0 & 0 & 0 & \lambda_{1} \lambda_{3} \\
0 & 0 & 0 & 0 & 0 & 0 & 0 & 0 & 0 \\
0 & 0 & 0 & 0 & 0 & 0 & 0 & 0 & 0 \\
0 & 0 & 0 & 0 & 0 & 0 & 0 & 0 & 0 \\
0 & 0 & 0 & 0 & 0 & 0 & 0 & 0 & 0 \\
0 & 0 & 0 & 0 & 0 & 0 & 0 & 0 & 0 \\
0 & 0 & 0 & 0 & 0 & 0 & 0 & 0 & 0 \\
0 & 0 & 0 & 0 & 0 & 0 & 0 & 0 & 0 \\
\lambda_{1} \lambda_{3} & 0 & 0 & 0 & 0 & 0 & 0 & 0 & \lambda_{1}^{2}
\end{array}\right) .
$$

We select the Bell operator in (18) to be

$$
\mathcal{B}_{22}=\tilde{A}_{1}^{2} \otimes \tilde{B}_{1}^{2}+\tilde{A}_{1}^{2} \otimes \tilde{B}_{2}^{2}+\tilde{A}_{2}^{2} \otimes \tilde{B}_{1}^{2}-\tilde{A}_{2}^{2} \otimes \tilde{B}_{2}^{2},
$$

where $\tilde{A}_{k}^{2}=L_{2}^{A} A_{k}^{2}\left(L_{2}^{A}\right)^{\dagger}, \tilde{B}_{l}^{2}=L_{2}^{B} B_{l}^{2}\left(L_{2}^{B}\right)^{\dagger}$, and

$$
\begin{gathered}
A_{k}^{2}=\left(\begin{array}{ccc}
-a_{k}^{3} & 0 & a_{k}^{1}+a_{k}^{2} i \\
0 & 0 & 0 \\
a_{k}^{1}-a_{k}^{2} i & 0 & a_{k}^{3}
\end{array}\right), \\
B_{l}^{1}=\left(\begin{array}{ccc}
-b_{l}^{3} & 0 & b_{l}^{1}+b_{l}^{2} i \\
0 & 0 & 0 \\
b_{l}^{1}-b_{l}^{2} i & 0 & b_{l}^{3}
\end{array}\right), k, l=1,2 .
\end{gathered}
$$

We then obtain the maximal violation of the inequality (19): $2 \sqrt{1+\frac{4 \lambda_{1}^{2} \lambda_{3}^{2}}{\left(\lambda_{1}^{2}+\lambda_{3}^{2}\right)^{2}}}>2$.

\subsection{Inequalities for multipartite quantum systems}

We now consider multipartite quantum systems. For convenience we consider that all subsystems have the same dimensions. However, as seen in the following, our discussions also apply to multipartite quantum systems with different dimensions.

Let $H$ denote a $d$-dimensional vector space with basis $|i\rangle$, where $i=1,2, \ldots, d$. An $L$-partite pure state in $H \otimes \cdots \otimes H$ is generally of the form

$$
|\Psi\rangle=\sum_{i_{1}, i_{2}, \cdots i_{L}=1}^{d} a_{i_{1}, i_{2}, \cdots i_{L}}\left|i_{1}, i_{2}, \cdots i_{L}\right\rangle, a_{i_{1}, i_{2}, \cdots i_{L}} \in \mathbb{C} .
$$

Let $\alpha$ and $\alpha^{\prime}$ (resp. $\beta$ and $\beta^{\prime}$ ) be subsets of the subindices of $a$, associated with the same sub-vector spaces but having different summing indices. $\alpha$ (or $\alpha^{\prime}$ ) and $\beta$ (or $\beta^{\prime}$ ) span the whole space of the given sub-index of $a$. A possible combination of the indices of $\alpha$ and $\beta$ can be considered as a kind of bipartite decomposition of the $L$ subsystems, say part A and part B, containing $m$ and $n=L-m$ subsystems respectively.

For a given bipartite decomposition, we can employ analysis similar to the bipartite case. Let $L_{\alpha}^{A}$ and $L_{\beta}^{B}$ be the generators of special unitary groups $S O\left(d^{m}\right)$ and $S O\left(d^{n}\right)$. By projecting $|\Psi\rangle$ onto $2 \times 2$ subsystems, we have "two-qubit" pure states:

$$
\rho_{\alpha \beta}^{p}=\frac{L_{\alpha}^{A} \otimes L_{\beta}^{B} \rho\left(L_{\alpha}^{A}\right)^{\dagger} \otimes\left(L_{\beta}^{B}\right)^{\dagger}}{\left\|L_{\alpha}^{A} \otimes L_{\beta}^{B} \rho\left(L_{\alpha}^{A}\right)^{\dagger} \otimes\left(L_{\beta}^{B}\right)^{\dagger}\right\|},
$$

where $\alpha=1,2, \cdots, \frac{d^{m}\left(d^{m}-1\right)}{2}, \beta=1,2, \cdots, \frac{d^{n}\left(d^{n}-1\right)}{2}$, and $p$ is the bipartite decomposition of the $L$ subsystems.

For each pure state $\rho_{\alpha \beta}^{p}$ we define the corresponding Bell operators

$$
\mathcal{B}_{\alpha \beta}^{p}=\tilde{A}_{1}^{\alpha} \otimes \tilde{B}_{1}^{\beta}+\tilde{A}_{1}^{\alpha} \otimes \tilde{B}_{2}^{\beta}+\tilde{A}_{2}^{\alpha} \otimes \tilde{B}_{1}^{\beta}-\tilde{A}_{2}^{\alpha} \otimes \tilde{B}_{2}^{\beta},
$$

where $\tilde{A}_{i}^{\alpha}=L_{\alpha}^{A} A_{i}^{\alpha}\left(L_{\alpha}^{A}\right)^{\dagger}$ and $\tilde{B}_{j}^{\beta}=L_{\beta}^{B} B_{j}^{\beta}\left(L_{\beta}^{B}\right)^{\dagger}$ are the Hermitian operators similarly defined as in (18).

Theorem 2 Any multipartite pure quantum state is entangled if and only if at least one of the following inequalities is violated [36]:

$$
\left|\left\langle\mathcal{B}_{\alpha \beta}^{p}\right\rangle\right| \leqslant 2 .
$$

Proof Obviously, multipartite quantum states that violate any one of the Bell inequalities in (27) must be entangled.

We now prove that, for any entangled multipartite pure quantum state, at least one of the inequalities in (27) is violated. The concurrence of $|\Psi\rangle$ is given by [41]

$$
C_{d}^{L}(|\Psi\rangle)=\sqrt{K \sum_{p} \sum_{\left\{\alpha, \alpha^{\prime}, \beta, \beta^{\prime}\right\}}^{d}\left|a_{\alpha \beta} a_{\alpha^{\prime} \beta^{\prime}}-a_{\alpha \beta^{\prime}} a_{\alpha^{\prime} \beta}\right|^{2},}
$$

where $K=d / 2 m(d-1), m=2^{L-1}-1$, and $\sum_{p}$ is the summation over all possible combinations of the indices of $\alpha$ and $\beta$. (28) can be rewritten as

$$
C_{d}^{L}(|\Psi\rangle)=\sqrt{K \sum_{p} \sum_{\alpha \beta}\left(C\left(\rho_{\alpha \beta}^{p}\right)\right)^{2}},
$$

where $\rho_{\alpha \beta}^{p}$ are defined in (25). As $|\Psi\rangle$ is an entangled state, $C(|\Psi\rangle)$ must be nonzero: i.e. at least one of $\rho_{\alpha \beta}^{p}$, say $\rho_{\alpha_{0} \beta_{0}}^{p_{0}}$, has nonzero concurrence. As discussed above, $\rho_{\alpha_{0} \beta_{0}}^{p_{0}}$ is actually a two-qubit quantum pure state. An entangled two-qubit quantum pure state must violate the Bell inequality (3).

As an example, we consider three-qubit systems. Acin et al. verified that any general pure three-qubit state $|\Psi\rangle$ can be uniquely written as [42]

$|\Psi\rangle=\lambda_{0}|000\rangle+\lambda_{1} e^{i \psi}|100\rangle+\lambda_{2}|101\rangle+\lambda_{3}|110\rangle+\lambda_{4}|111\rangle$,

where $\lambda_{i} \geqslant 0,0 \leqslant \psi \leqslant \pi$, and $\sum_{i} \lambda_{i}^{2}=1$. From straightforward mathematics, we have

$$
C^{2}(|\Psi\rangle)=2\left(\lambda_{0} \lambda_{2}\right)^{2}+2\left(\lambda_{0} \lambda_{4}\right)^{2}+\left|2 e^{i \psi} \lambda_{1} \lambda_{4}-2 \lambda_{2} \lambda_{3}\right|^{2}
$$




$$
\begin{aligned}
& +2\left(\lambda_{0} \lambda_{3}\right)^{2}+2\left(\lambda_{0} \lambda_{4}\right)^{2}+\left|2 e^{i \psi} \lambda_{1} \lambda_{4}-2 \lambda_{2} \lambda_{3}\right|^{2} \\
& +2\left(\lambda_{0} \lambda_{2}\right)^{2}+2\left(\lambda_{0} \lambda_{3}\right)^{2}+2\left(\lambda_{0} \lambda_{4}\right)^{2} .
\end{aligned}
$$

We now give a detailed analysis of how the entangled pure three-qubit state( i.e. at least one of the terms on the right hand side of (31) is non-zero) must violate one of the inequalities in (27).

Case 1 If $\lambda_{0} \lambda_{2} \neq 0$, the corresponding operator $L_{2}^{A} \otimes L_{1}^{B}=$ $\left(\begin{array}{cccc}0 & 0 & 1 & 0 \\ 0 & 0 & 0 & 0 \\ -1 & 0 & 0 & 0 \\ 0 & 0 & 0 & 0\end{array}\right) \otimes\left(\begin{array}{cc}0 & 1 \\ -1 & 0\end{array}\right)$ and $\rho_{21}^{12 \mid 3}=\left(\begin{array}{cccccccc}\lambda_{2}^{2} & -e^{-i \psi} \lambda_{1} \lambda_{2} & 0 & 0 & 0 & \lambda_{0} \lambda_{2} & 0 & 0 \\ -e^{i \psi} \lambda_{1} \lambda_{2} & \lambda_{1}^{2} & 0 & 0 & 0 & -e^{i \psi} \lambda_{0} \lambda_{1} & 0 & 0 \\ 0 & 0 & 0 & 0 & 0 & 0 & 0 & 0 \\ 0 & 0 & 0 & 0 & 0 & 0 & 0 & 0 \\ 0 & 0 & 0 & 0 & 0 & 0 & 0 & 0 \\ \lambda_{0} \lambda_{2} & -e^{-i \psi} \lambda_{0} \lambda_{1} & 0 & 0 & 0 & \lambda_{0}^{2} & 0 & 0 \\ 0 & 0 & 0 & 0 & 0 & 0 & 0 & 0 \\ 0 & 0 & 0 & 0 & 0 & 0 & 0 & 0\end{array}\right)$.

We choose the Bell operator in (26) to be that with respect to the bipartite decomposition of the first two qubits and the last one:

$$
\mathcal{B}_{21}^{12 \mid 3}=\tilde{A}_{1}^{2} \otimes \tilde{B}_{1}^{1}+\tilde{A}_{1}^{2} \otimes \tilde{B}_{2}^{1}+\tilde{A}_{2}^{2} \otimes \tilde{B}_{1}^{1}-\tilde{A}_{2}^{2} \otimes \tilde{B}_{2}^{1},
$$

where $\tilde{A}_{k}^{2}=L_{2}^{A} A_{k}^{2}\left(L_{2}^{A}\right)^{\dagger}, \quad \tilde{B}_{l}^{1}=L_{1}^{B} B_{l}^{1}\left(L_{1}^{B}\right)^{\dagger}$, and $A_{k}^{2}=$ $\left(\begin{array}{cccc}-a_{k}^{3} & 0 & a_{k}^{1}+a_{k}^{2} i & 0 \\ 0 & 0 & 0 & 0 \\ a_{k}^{1}-a_{k}^{2} i & 0 & a_{k}^{3} & 0 \\ 0 & 0 & 0 & 0\end{array}\right), B_{l}^{1}=\left(\begin{array}{cc}-b_{l}^{3} & b_{l}^{1}+b_{l}^{2} i \\ b_{l}^{1}-b_{l}^{2} i & b_{l}^{3}\end{array}\right)$ with $k, l=1,2$. We thus have the maximal violation of the inequality (27), $2 \sqrt{1+\frac{4 \lambda_{0}^{2} \lambda_{2}^{2}}{\left(\lambda_{0}^{2}+\lambda_{1}^{2}+\lambda_{2}^{2}\right)^{2}}}>2$.

Case 2 If $\left|e^{i \psi} \lambda_{1} \lambda_{4}-\lambda_{2} \lambda_{3}\right| \neq 0$, the corresponding operator is $L_{6}^{A} \otimes L_{1}^{B}=\left(\begin{array}{cccc}0 & 0 & 0 & 0 \\ 0 & 0 & 0 & 0 \\ 0 & 0 & 0 & 1 \\ 0 & 0 & -1 & 0\end{array}\right) \otimes\left(\begin{array}{cc}0 & 1 \\ -1 & 0\end{array}\right)$. The matrix $\rho_{61}^{12 \mid 3}$ has only nonzero entries at the right down corner with the form,

$$
\left(\begin{array}{cccc}
\lambda_{4}^{2} & -\lambda_{3} \lambda_{4} & -\lambda_{2} \lambda_{4} & e^{-i \psi} \lambda_{1} \lambda_{4} \\
-\lambda_{3} \lambda_{4} & \lambda_{3}^{2} & \lambda_{2} \lambda_{3} & -e^{-i \psi} \lambda_{1} \lambda_{3} \\
-\lambda_{2} \lambda_{4} & \lambda_{2} \lambda_{3} & \lambda_{2}^{2} & -e^{-i \psi} \lambda_{1} \lambda_{2} \\
e^{i \psi} \lambda_{1} \lambda_{4} & -e^{i \psi} \lambda_{1} \lambda_{3} & -e^{i \psi} \lambda_{1} \lambda_{2} & \lambda_{1}^{2}
\end{array}\right)
$$

The Bell operator in (26) has the form

$$
\mathcal{B}_{61}^{12 \mid 3}=\tilde{A}_{1}^{6} \otimes \tilde{B}_{1}^{1}+\tilde{A}_{1}^{6} \otimes \tilde{B}_{2}^{1}+\tilde{A}_{2}^{6} \otimes \tilde{B}_{1}^{1}-\tilde{A}_{2}^{6} \otimes \tilde{B}_{2}^{1},
$$

where $\tilde{A}_{k}^{6}=L_{6}^{A} A_{k}^{6}\left(L_{6}^{A}\right)^{\dagger}, \quad \tilde{B}_{l}^{1}=L_{1}^{B} B_{l}^{1}\left(L_{1}^{B}\right)^{\dagger}$, and $A_{k}^{6}=$ $\left(\begin{array}{cccc}0 & 0 & 0 & 0 \\ 0 & 0 & 0 & 0 \\ 0 & 0 & -a_{k}^{3} & a_{k}^{1}+a_{k}^{2} i \\ 0 & 0 & a_{k}^{1}-a_{k}^{2} i & a_{k}^{3}\end{array}\right), B_{l}^{1}=\left(\begin{array}{cc}-b_{l}^{3} & b_{l}^{1}+b_{l}^{2} i \\ b_{l}^{1}-b_{l}^{2} i & b_{l}^{3}\end{array}\right)$, $k, l=1,2$. The corresponding maximal violation is given by $2 \sqrt{1+\frac{4\left|e^{i \psi} \lambda_{1} \lambda_{4}-\lambda_{2} \lambda_{3}\right|^{2}}{\left(\lambda_{1}^{2}+\lambda_{2}^{2}+\lambda_{3}^{2}+\lambda_{4}^{2}\right)^{2}}}$, which is obviously strictly larger than 2. Other cases can be discussed similarly.

Nevertheless, (27) is not yet a Bell-type inequality in the usual sense, because we treated the problem by considering all possible bipartite decompositions. Generally it only services as a sufficient and necessary condition for separability of multipartite pure states. For some particular cases, the operators (26) for multipartite systems become local ones as in the standard Bell inequalities. For instance, the Bell operator (32) in the example can be written in a very simple form:

$$
\begin{aligned}
\mathcal{B}_{61}^{12 \mid 3} & =\tilde{A}_{1}^{6} \otimes \tilde{B}_{1}^{1}+\tilde{A}_{1}^{6} \otimes \tilde{B}_{2}^{1}+\tilde{A}_{2}^{6} \otimes \tilde{B}_{1}^{1}-\tilde{A}_{2}^{6} \otimes \tilde{B}_{2}^{1} \\
& =\left(\frac{I-\sigma^{z}}{2}\right) \otimes\left(\tilde{A}^{\prime}{ }_{1}^{6} \otimes \tilde{B}_{1}^{1}+{\tilde{A^{\prime}}}_{1}^{6} \otimes \tilde{B}_{2}^{1}+{\tilde{A^{\prime}}}_{2}{ }_{2}^{6} \otimes \tilde{B}_{1}^{1}-{\tilde{A^{\prime}}}_{2}^{6} \otimes \tilde{B}_{2}^{1}\right),
\end{aligned}
$$

where ${\tilde{A^{\prime}}}^{\prime}{ }_{k}=\left(\begin{array}{cc}a_{k}^{3} & -a_{k}^{1}+i a_{k}^{2} \\ -a_{k}^{1}-i a_{k}^{2} & -a_{k}^{3}\end{array}\right)$, with $k=1,2$. Therefore, our Bell operators acting on multipartite states can be expressed as real linear combinations of local operators in this case.

\subsection{Bell inequalities and distillation}

A bipartite state $\rho$ is called distillable, if and only if maximally entangled bipartite pure states (e.g. $\left|\psi_{+}\right\rangle=\frac{1}{\sqrt{2}}(|00\rangle+$ $|11\rangle)$ ) can be created from a number of identical copies of the state $\rho$ by means of local operations and classical communication. We call a multipartite state distillable if and only if there exists at least one bipartite decomposition of the system such that pure entangled states can be distilled. It has been shown that all quantum entangled pure states are distillable. However, it is a challenge to give an operational criterion of distillability for general mixed states. A sufficient condition of distillability has been presented [37]. Our inequalities (27) are both sufficient and necessary for the separability of pure states, but generally not for the separability of mixed states. However, surprisingly (27) can serve as a criterion for distillability.

Theorem 3 Any bipartite quantum state $\rho$ that violates any one of the Bell inequalities in (19) (i.e. $\operatorname{Tr}\left\{\mathcal{B}_{\alpha \beta} \rho\right\}>2$ ) is always distillable. In addition, if a multipartite quantum state $\rho$ violates one of the Bell inequalities in (27) (i.e. $\rho$ satisfies 
$\operatorname{Tr}\left\{\mathcal{B}_{\alpha \beta}^{p} \rho\right\}>2$ ), then bipartite maximally entangled pure states can be distilled from the copies of $\rho$.

Proof It has been shown that a density matrix $\rho$ is distillable if and only if there are projectors $P$, and $Q$ that map high-dimensional spaces to two-dimensional spaces such that the state $P \otimes Q \rho^{\otimes s} P \otimes Q$ is entangled for some $s$ copies [43]. Thus if any one of the Bell inequalities in (19) is violated, there exists a submatrix $\rho_{\alpha \beta}$, like (20), that has nonzero concurrence. For a generally given operator $L_{\alpha}=|i\rangle\langle j|-| j\rangle\langle i|$, $L_{\beta}=|k\rangle\langle l|-| l\rangle\langle k|$, the operators $P$ and $Q$ are explicitly given by $P=A L_{\alpha}$ and $Q=B L_{\beta}$, where $A=\left|0_{A}\right\rangle\left\langle i|+| 1_{A}\right\rangle\langle j|$, $B=\left|0_{B}\right\rangle\left\langle k|+| 1_{B}\right\rangle\left\langle l|,| 0_{A / B}\right\rangle$ and $\left|1_{A / B}\right\rangle$ are the orthonormal bases of a two-dimensional vector space. $P \otimes Q$ maps state $\rho$ to a two-qubit state that has the same nonzero concurrence as $\rho_{\alpha \beta}$. Since any entangled two-qubit state is distillable, $\rho$ is distillable. The multipartite case can be discussed similarly.

Remark It has been shown that positive partial transposition (PPT) entangled quantum states are not distillable $[44,45]$. Therefore PPT quantum states should never violate the Bell inequalities in (19) or (27). This can be seen from the following. A density matrix $\rho$ is said to have a PPT property if the partial transposition of $\rho$ with respect to any subsystem(s) is still positive. Let $\rho^{T_{B}}$ denote the partial transposition with respect to the subsystem $B$. Assume that there is a PPT state $\rho$ violating one of the Bell inequalities in (27), say $\operatorname{Tr}\left\{\mathcal{B}_{\alpha_{0} \beta_{0}}^{p_{0}} \rho\right\}>2$. This can be equivalently understood as there exists two-qubit state $\rho_{\alpha_{0} \beta_{0}}^{p_{0}}$ in the form of (25) such that $\operatorname{Tr}\left\{B_{\alpha_{0} \beta_{0}}^{p_{0}} \rho_{\alpha_{0} \beta_{0}}^{p_{0}}\right\}>2$, where $B_{\alpha_{0} \beta_{0}}^{p_{0}}=$ $A_{1}^{\alpha_{0}} \otimes B_{1}^{\beta_{0}}+A_{1}^{\alpha_{0}} \otimes B_{2}^{\beta_{0}}+A_{2}^{\alpha_{0}} \otimes B_{1}^{\beta_{0}}-A_{2}^{\alpha_{0}} \otimes B_{2}^{\beta_{0}}$. On the other hand, using the PPT property of $\rho$, we have

$$
\rho_{\alpha_{0} \beta_{0}}^{T_{B}}=L_{\alpha_{0}}^{A} \otimes\left(L_{\beta_{0}}^{B}\right)^{*} \rho^{T_{B}}\left(L_{\alpha_{0}}^{A}\right)^{\dagger} \otimes\left(L_{\beta_{0}}^{B}\right)^{T} \geqslant 0 .
$$

As both $L_{\alpha_{0}}^{A}$ and $L_{\beta_{0}}^{B}$ are projectors to two-dimensional subspaces, $\rho_{\alpha_{0} \beta_{0}}^{p_{0}}$ can be considered as a $2 \times 2$ state. While a $2 \times 2$ PPT state $\rho_{\alpha_{0} \beta_{0}}$ must be separable [46], it contradicts with $\operatorname{Tr}\left\{B_{\alpha_{0} \beta_{0}}^{p_{0}} \rho_{\alpha_{0} \beta_{0}}^{p_{0}}\right\}>2$.

\section{Bell inequalities detecting more (mixed) entangled bipartite states}

We now consider bipartite states for $N \times N$ systems. For even $N$, let $\Gamma_{x}, \Gamma_{y}$ and $\Gamma_{z}$ be block-diagonal matrices in which each block is an ordinary Pauli matrix, $\sigma_{x}, \sigma_{y}$ and $\sigma_{z}$ repectively, as described in [17] for $\Gamma_{x}$ and $\Gamma_{z}$. When $N$ is odd, we set the elements of the $k$ th row and the $k$ th column in $\Gamma_{x}, \Gamma_{y}$ and $\Gamma_{z}$ to be zero. The other elements of $\Gamma_{x}, \Gamma_{y}$ and $\Gamma_{z}$ are the blockdiagonal matrices as for the even- $N$ case. Let $\Pi(k)$ be an $N \times N$ matrix whose only nonvanishing entry is $(\Pi(k))_{k k}=1$, $k \in 1,2, \cdots, N$, for odd $N$ and be a null matrix for even $N$. We define observables

$$
A=\vec{a} \cdot \vec{\Gamma}+\Pi(k)=a_{x} \Gamma_{x}+a_{y} \Gamma_{y}+a_{z} \Gamma_{z}+\Pi(k)
$$

and

$$
B=\vec{b} \cdot \vec{\Gamma}+\Pi(k)=b_{x} \Gamma_{x}+b_{y} \Gamma_{y}+b_{z} \Gamma_{z}+\Pi(k),
$$

where $\vec{a}=\left(a_{x}, a_{y}, a_{z}\right)$ and $\vec{b}=\left(b_{x}, b_{y}, b_{z}\right)$ are real unit vectors

We define the Bell operator as [38]

$$
\mathcal{B}=A_{1} \otimes B_{1}+A_{1} \otimes B_{2}+A_{2} \otimes B_{1}-A_{2} \otimes B_{2},
$$

where

$$
\begin{aligned}
A_{i} & =\vec{a}_{i} \cdot \vec{\Gamma}+\Pi(k)=a_{i}^{x} \Gamma_{x}+a_{i}^{y} \Gamma_{y}+a_{i}^{z} \Gamma_{z}+\Pi(k), \\
B_{i} & =\vec{b}_{j} \cdot \vec{\Gamma}+\Pi(k)=b_{j}^{x} \Gamma_{x}+b_{j}^{y} \Gamma_{y}+b_{j}^{z} \Gamma_{z}+\Pi(k) .
\end{aligned}
$$

Theorem 4 If there exists a local hidden-variable model to describe the system, the inequality

$$
|\langle\mathcal{B}\rangle| \leqslant 2
$$

must hold for any $\vec{a}_{i}, \vec{b}_{i}, i=1,2$, and all $k \in 1,2, \cdots, N$.

The proof of this theorem is straightforward. Note that for any three-dimensional unit vectors $\vec{a}$ and $\vec{b}$, the eigenvalues of $A$ and $B$ are either 1 or -1 . Then as discussed for the two-qubit case, if there exists a local hidden-variable model to describe the system, we have

$$
\begin{aligned}
|\langle\mathcal{B}\rangle| & =\left|\left\langle A_{1} \otimes B_{1}+A_{1} \otimes B_{2}+A_{2} \otimes B_{1}-A_{2} \otimes B_{2}\right\rangle\right| \\
& =\left|\left\langle A_{1} \otimes\left(B_{1}+B_{2}\right)\right\rangle+\left\langle A_{2} \otimes\left(B_{1}-B_{2}\right)\right\rangle\right| \\
& \leqslant\left|\left\langle A_{1}\right\rangle\right|\left|\left\langle\left(B_{1}+B_{2}\right)\right\rangle\right|+\left|\left\langle A_{2}\right\rangle\right|\left|\left\langle\left(B_{1}-B_{2}\right)\right\rangle\right| \leqslant 2 .
\end{aligned}
$$

We now compute the maximal violation of the Bell inequality.

Proposition 1 For any bipartite pure state $|\psi\rangle$ with even $N$, the maximal violation of the Bell inequality (37) is given by [38]

$$
\max \langle\psi|\mathcal{B}| \psi\rangle=2 \sqrt{\tau_{1}+\tau_{2}},
$$

where $\tau_{1}$ and $\tau_{2}$ are the two largest eigenvalues of the matrix $R^{T} R, R$ is the matrix with entries $R_{\alpha \beta}=\left\langle\psi\left|\Gamma_{\alpha} \otimes \Gamma_{\beta}\right| \psi\right\rangle$, with $\alpha, \beta=x, y, z$.

Proof If $N$ is even, we have the maximal violation of the Bell inequalities (37)

$$
\begin{aligned}
\max \langle\psi|\mathcal{B}| \psi\rangle= & \max _{\vec{a}_{1}, \vec{a}_{2}, \vec{b}_{1}, \vec{b}_{2}}\left[\left\langle\psi\left|\sum_{\alpha=x, y, z} a_{1}^{\alpha} \Gamma_{\alpha} \otimes \sum_{\beta=x, y, z}\left(b_{1}^{\beta}+b_{2}^{\beta}\right) \Gamma_{\beta}\right| \psi\right\rangle\right. \\
& \left.+\left\langle\psi\left|\sum_{\alpha=x, y, z} a_{2}^{\alpha} \Gamma_{\alpha} \otimes \sum_{\beta=x, y, z}\left(b_{1}^{\beta}-b_{2}^{\beta}\right) \Gamma_{\beta}\right| \psi\right\rangle\right] \\
= & \max _{\vec{a}_{1}, \vec{a}_{2}, \vec{b}_{1}, \vec{b}_{2}}\left[\vec{a}_{1} \cdot R\left(\vec{b}_{1}+\vec{b}_{2}\right)+\vec{a}_{2} \cdot R\left(\vec{b}_{1}-\vec{b}_{2}\right)\right] \\
= & \max _{\vec{b}_{1}, \vec{b}_{2}}\left[\left\|R\left(\vec{b}_{1}+\vec{b}_{2}\right)\right\|+\left\|R\left(\vec{b}_{1}-\vec{b}_{2}\right)\right\|\right] \\
= & \max _{\theta, \vec{c} \perp \vec{c}^{\prime}} 2\left[\cos \theta\|R \vec{c}\|+\sin \theta\left\|R \vec{c}^{\prime}\right\|\right] \\
= & \max _{\vec{c} \perp \vec{c}^{\prime}} 2 \sqrt{\|R \vec{c}\|^{2}+\left\|R \vec{c}^{\prime}\right\|^{2}}=2 \sqrt{\tau_{1}+\tau_{2}},
\end{aligned}
$$

where $\vec{a}_{i}=\left(a_{i}^{x}, a_{i}^{y}, a_{i}^{z}\right), \vec{b}_{j}=\left(b_{j}^{x}, b_{j}^{y}, b_{j}^{z}\right), i, j=1,2$. 
Proposition 2 For any bipartite pure state $|\Psi\rangle$ in the Schmidt bi-orthogonal form,

$$
|\Psi\rangle=\sum_{i=1}^{N} c_{i}|i i\rangle, \quad c_{i} \in R, \quad \sum_{i} c_{i}^{2}=1
$$

with odd $N$, the maximal violation of the Bell inequality (37) is given by [38]

$$
\max \langle\Psi|\mathcal{B}| \Psi\rangle=2 \sqrt{\tau_{1}+\tau_{2}}+2\langle\Psi|\Pi(k) \otimes \Pi(k)| \Psi\rangle,
$$

where $\tau_{1}$ and $\tau_{2}$ are defined in Proposition 1 .

Proof For odd $N$ and any $k \in\{1,2, \cdots, N\}$, similarly we have

$$
\begin{aligned}
& \max \langle\Psi|B| \Psi\rangle=\max _{\vec{a}_{1}, \vec{a}_{2}, \vec{b}_{1}, \vec{b}_{2}}\left[\langle\Psi|\left(\sum_{\alpha=x, y, z} a_{1}^{\alpha} \Gamma_{\alpha}+\Pi(k)\right)\right. \\
& \otimes\left(\sum_{\beta=x, y, z}\left(b_{1}^{\beta}+b_{2}^{\beta}\right) \Gamma_{\beta}+2 \Pi(k)\right)|\Psi\rangle \\
& +\langle\Psi|\left(\sum_{\alpha=x, y, z} a_{2}^{\alpha} \Gamma_{\alpha}+\Pi(k)\right) \\
& \left.\otimes\left(\sum_{\beta=x, y, z}\left(b_{1}^{\beta}-b_{2}^{\beta}\right) \Gamma_{\beta}\right)|\Psi\rangle\right] \\
& =\max _{\vec{a}_{1}, \vec{a}_{2}, \vec{b}_{1}, \vec{b}_{2}}\left[\vec{a}_{1} \cdot R\left(\vec{b}_{1}+\vec{b}_{2}\right)+\vec{a}_{2} \cdot R\left(\vec{b}_{1}-\vec{b}_{2}\right)\right] \\
& +2\langle\Psi|\Pi(k) \otimes \Pi(k)| \Psi\rangle \\
& =\max _{\vec{b}_{1}, \vec{b}_{2}}\left[\left\|R\left(\vec{b}_{1}+\vec{b}_{2}\right)\right\|+\left\|R\left(\vec{b}_{1}-\vec{b}_{2}\right)\right\|\right] \\
& +2\langle\Psi|\Pi(k) \otimes \Pi(k)| \Psi\rangle \\
& =\max _{\theta, \vec{c} \perp \vec{c}} 2\left[\cos \theta\|R \vec{c}\|+\sin \theta\left\|R \vec{c}^{\prime}\right\|\right] \\
& +2\langle\Psi|\Pi(k) \otimes \Pi(k)| \Psi\rangle \\
& =\max _{\vec{c} \perp \vec{c}^{\prime}} 2 \sqrt{\|R \vec{c}\|^{2}+\left\|R \overrightarrow{c^{\prime}}\right\|^{2}}+2\langle\Psi|\Pi(k) \otimes \Pi(k)| \Psi\rangle \\
& =2 \sqrt{\tau_{1}+\tau_{2}}+2\langle\Psi|\Pi(k) \otimes \Pi(k)| \Psi\rangle .
\end{aligned}
$$

Remark For even $N$, formula (38) is also valid for any bipartite mixed quantum state $\rho$. One only needs to redefine $R_{\alpha \beta}=\operatorname{Tr}\left[\rho \Gamma_{\alpha} \otimes \Gamma_{\beta}\right]$ for $\alpha, \beta=x, y, z$. Formula (40) does not fit for general quantum states with odd $N$. However, for some quantum mixed states the maximal violation of the Bell inequality (37) can still be computed using the formula (see example 2 below).

Moreover, the Bell inequality in [17] is a special case of (37) in the sense that it can be obtained by setting $a_{y}$ and $b_{y}$ in (34) and (35) to be zero, and $k=N$ in the Bell operator (36). For $k=N$, the maximal violation of (37) for an arbitrary bipartite quantum state (39) is the same as the violation values given in [17]. This means that the parameters $a_{y}$ and $b_{y}$ do not contribute to the maximal violation in this case. However, even in this case the formulae (38) and (40) have their own advantages. On one hand, one can compute the maximal violation without choosing proper Bell operator as is needed in [17]. On the other hand, for odd $N$, more entangled quantum states can be detected by adjusting $k$. In the following we give two examples to illustrate these properties.

Example 1 Consider a $3 \times 3$ pure state with Schmidt decomposition $|\psi\rangle=(|11\rangle+|33\rangle) / \sqrt{2}$. Using the Bell operator given in [17] we obtain the maximal violation 2, which fails to detect the entanglement. Now taking $k=2$ we obtain the maximal violation of the Bell inequality (37) $2 \sqrt{2}$, which means that $|\psi\rangle$ is entangled.

The Bell inequality (37) is valid also for all mixed states with even $N$ and for some mixed states with odd $N$. Therefore it can be used to detect experimentally the entanglement of mixed states.

Example 2 Consider the maximally entangled state $\left|\psi_{+}\right\rangle=\sum_{i=1}^{N} \frac{1}{\sqrt{N}}|i i\rangle$ mixed with noise:

$$
\rho(x)=\frac{x}{N^{2}} I+(1-x)\left|\psi_{+}\right\rangle\left\langle\psi_{+}\right| .
$$

For even $N$, the maximal violation of $\rho(x)$ is $2 \sqrt{2}(1-x)$. Therefore, the Bell inequality (37) detects entanglement of $\rho(x)$ for $0 \leqslant x<0.292893$. If $N$ is odd, we note that for any $k \in\{1,2, \cdots, N\}$ and $\alpha \in\{x, y, z\},\left(\Gamma_{\alpha}\right)_{k k}=0$. Thus we have

$$
\operatorname{Tr}\left[\rho(x)\left(\Gamma_{\alpha} \otimes \Pi(k)\right)\right]=\operatorname{Tr}\left[\rho(x)\left(\Pi(k) \otimes \Gamma_{\alpha}\right)\right]=0 .
$$

Taking into account (42) we have the maximal violation

$$
\begin{aligned}
\max \operatorname{Tr}[\rho(x) B]= & \max _{\vec{a}_{1}, \vec{a}_{2}, \vec{b}_{1}, \vec{b}_{2}}\left\{\operatorname { T r } \left[\rho(x)\left(\sum_{\alpha=x, y, z} a_{1}^{\alpha} \Gamma_{\alpha}+\Pi(k)\right)\right.\right. \\
& \left.\otimes\left(\sum_{\beta=x, y, z}\left(b_{1}^{\beta}+b_{2}^{\beta}\right) \Gamma_{\beta}+2 \Pi(k)\right)\right] \\
& +\operatorname{Tr}\left[\rho(x)\left(\sum_{\alpha=x, y, z} a_{2}^{\alpha} \Gamma_{\alpha}+\Pi(k)\right)\right. \\
& \left.\left.\otimes\left(\sum_{\beta=x, y, z}\left(b_{1}^{\beta}-b_{2}^{\beta}\right) \Gamma_{\beta}\right)\right]\right\} \\
= & \max _{\vec{a}_{1}, \vec{a}_{2}, \vec{b}_{1}, \vec{b}_{2}}\left[\vec{a}_{1} \cdot R\left(\vec{b}_{1}+\vec{b}_{2}\right)+\vec{a}_{2} \cdot R\left(\vec{b}_{1}-\vec{b}_{2}\right)\right] \\
& +2 \operatorname{Tr}[\rho(x) \Pi(k) \otimes \Pi(k)] \\
= & \max _{\vec{c} \perp \vec{c}^{\prime}} 2 \sqrt{\|R \vec{c}\|^{2}+\left\|R \vec{c}^{\prime}\right\|^{2}} \\
& +2 \operatorname{Tr}[\rho(x) \Pi(k) \otimes \Pi(k)] \\
= & 2 \sqrt{\tau_{1}+\tau_{2}}+2 \operatorname{Tr}[\rho(x) \Pi(k) \otimes \Pi(k)],
\end{aligned}
$$

where $R_{\alpha \beta}=\operatorname{Tr}\left[\rho \Gamma_{\alpha} \otimes \Gamma_{\beta}\right]$. For $N=3$, the maximal violation of $\rho(x)$ is $\frac{2}{25}(5-4 x)+\frac{8 \sqrt{2}}{5}(1-x)$. Hence the Bell inequality (37) can detect the entanglement of $\rho(x)$ for $0 \leqslant x<0.2566$ in this case. 


\section{Conclusions and remarks}

In this review, we have introduced several kinds of Bell inequalities such as the CHSH, MABK, WWZB and CGLMP Bell inequalities that rule out the local hidden-variable theories and help detect quantum entanglement experimentally. We have also introduced a series of Bell inequalities for bipartite quantum states by projecting the whole quantum systems to "two-qubit" subsystems. It has been shown that quantum states violating any one of these Bell inequalities are entangled. On the other hand, any entangled pure quantum state must violate at least one of these Bell-like inequalities. It has also been shown that quantum mixed states that violate the Bell inequalities must be distillable.

By constructing new Bell operators bipartite Bell inequalities that include Gisin's Bell inequalities in [17] as a special case have been also been introduced. The maximal violation of these Bell inequalities for pure states in Schmidt forms has been obtained. The formulae of maximal violation are valid also for all pure and mixed quantum states in evendimensional bipartite systems and for some mixed states in odd-dimensional bipartite systems. The new Bell inequality has been shown to be capable of detecting quantum entanglement more effectively.

In regards to the quantum separability most Bell inequalities so far work only for pure states. In [47] the authors used the fact that the PPT criterion $[44,45]$ is both sufficient and necessary for the separability of two-qubit mixed states and presented a formula for the detection of all entangled twoqubit mixed states experimentally in principle. Nevertheless, generally for mixed states less has been known for Bell inequalities. Concerning the conceptual foundations of quantum mechanics, to avoid state dependence in ruling out the local hidden-variable model in experiments, some "loopholefree" Bell inequalities have also been investigated [48, 49].

This work was supported by the National Natural Science Foundation of China (10875081), Key Program of Beijing Municipal Eduction Commission (KZ200810028013) and Academic Human Resources Development in Institutions of Higher Learning (PHR201007107).

1 Einstein A, Podolsky B, Rosen N. Can quantum-mechanical description of physical reality be considered complete? Phys Rev, 1935, 47: 777-780

2 Bell J S. On the Einstein-Podolsky-Rosen Paradox. Physics, 1964, 1: 195-200

3 Brukner Č, Żukowski M, Zeilinger A. Quantum communication complexity protocol with two entangled qutrits. Phys Rev Lett, 2002, 89: 197901

4 Scarani V, Gisin N. Quantum communication between $N$ partners and Bell's inequalities. Phys Rev Lett, 2001, 87: 117901

5 Acín A, Gisin N, Scarani V. Security bounds in quantum cryptography using d-level systems. Quantum Inf Comput, 2003, 3: 563-580

6 Clauser J F, Horne M A, Shimony A, et al. Proposed experiment to test local hidden-variable theories. Phys Rev Lett, 1969, 23: 880-884
7 Mermin N D. Extreme quantum entanglement in a superposition of macroscopically distinct states. Phys Rev Lett, 1990, 65: 1838-1840

8 Ardehali M. Bell inequalities with a magnitude of violation that grows exponentially with the number of particles. Phys Rev A, 1992, 46: 5375-5378

9 Belinskii A V, Klyshko D N. Interference of light and Bell's theorem. Phys Usp, 1993, 36: 653-693

10 Werner R F, Wolf M M. All-multipartite Bell-correlation inequalities for two dichotomic observables per site. Phys Rev A, 2001, 64: 032112

11 Żukowski M, Brukner Č. Bells Theorem for general $N$-qubit states. Phys Rev Lett, 2002, 88: 210401

12 Żukowski M, Brukner Č, Laskowski W, et al. Do all pure entangled states violate Bells inequalities for correlation functions? Phys Rev Lett, 2002, 88: 210402

13 Chen K, Albeverio S, Fei S M. Two-setting Bell inequalities for many qubits. Phys Rev A, 2006, 74: 050101(R)

14 Wu Y C, Badziag P, Wiesniak M, et al. Extending Bell inequalities to more parties. Phys Rev A, 2008, 77: 032105

15 Collins D, Gisin N, Linden N, et al. Bell inequalities for arbitrarily high-dimensional systems. Phys Rev Lett. 2002, 88: 040404

16 Gisin N. Bell's inequality holds for all non-product states. Phys Lett A, 1991, 154: 201-202

17 Gisin N, Peres A. Maximal violation of Bell's inequality for arbitrarily large spin. Phys Lett A, 1992, 162: 15-17

18 Chen J L, Wu C F, Kwek L C, et al. Gisin's Theorem for three qubits Phys Rev Lett, 2004, 93: 140407

19 Ekert A K. Quantum cryptography based on Bells theorem. Phys Rev Lett, 1991, 67: 661-663

20 Acín A, Brunner N, Gisin N, et al. Device-independent security of quantum cryptography against collective attacks. Phys Rev Lett, 2007, 98: 230501

21 Horodecki R, Horodecki M, Horodecki P. Teleportation, Bell's inequalities and inseparability. Phys Lett A, 1996, 222: 21-25

22 Lee S, Joo J, Kim J. Teleportation capability, distillability, and nonlocality on three-qubit states. Phys Rev A, 2007, 76: 012311

23 Dür W. Multipartite bound entangled states that violate Bells inequality. Phys Rev Lett, 2001, 87: 230402

24 Acín A. Distillability, Bell inequalities, and multiparticle bound entanglement. Phys Rev Lett, 2002, 88: 027901

25 Acín A, Scarani V, Wolf M M. Bells inequalities and distillability in N-quantum-bit systems. Phys Rev A, 2002, 66: 042323

26 Braunstein S L, Mann A, Revzen M. Maximal violation of Bell inequalities for mixed states. Phys Rev Lett, 1992, 68: 3259-3261

27 Horodecki R, Horodecki P, Horodecki M. Violating Bell inequality by mixed spin- $\frac{1}{2}$ states: Necessary and sufficient condition. Phys Lett A, 1995, 200: 340-344

28 Pan J W, Bouwmeester D, Daniell M, et al. Experimental test of quantum nonlocality in three-photon Greenberger-Horne-Zeilinger entanglement. Nature, 2000, 403: 515-519

29 Sun B Z, Fei S M. Bell inequalities classifying biseparable three-qubit states. Phys Rev A, 2006, 74: 032335

30 Chen J L, Deng D L, Hu M G. Gisins theorem for two d-dimensional systems based on the Collins-Gisin-Linden-Masser-Popescu inequality. Phys Rev A, 2008, 77: 060306(R)

31 Fu L B. General correlation functions of the Clauser-Horne-ShimonyHolt inequality for arbitrarily high-dimensional systems. Phys Rev Lett, 2004, 92: 130404

32 Chen J L, Deng D L. Tight correlation-function Bell inequality for multipartite d-dimensional systems. Phys Rev A, 2009, 79: 012111

33 Gisin N. Bell inequality for arbitrary many settings of the analyzers Phys Lett A, 1999, 260: 1-3

34 Ji S W, Lee J Y, Lim J, et al. Multisetting Bell inequality for qudits. Phys Rev A, 2008, 78: 052103 
35 Son W, Lee J, Kim M S. Generic Bell inequalities for multipartite arbitrary dimensional systems. Phys Rev Lett, 2006, 96: 060406

36 Li M, Fei S M. Gisins Theorem for arbitrary dimensional multipartite states. Phys Rev Lett, 2010, 104: 240502

37 Ou Y C, Fan H, Fei S M. Proper monogamy inequality for arbitrary pure quantum states. Phys Rev A, 2008, 78: 01231

38 Li M, Fei S M, Li-Jost X Q. Bipartite Bell inequality and maximal violation. Commun Theor Phys, 2011, 55: 418-420

39 Uhlmann A. Fidelity and concurrence of conjugated states. Phys Rev A, 2000, 62: 032307

40 Rungta P, Buz̈ek V, Caves C M, et al. Universal state inversion and concurrence in arbitrary dimensions. Phys Rev A, 2001, 64: 042315

41 Albeverio S, Fei S M. A note on invariants and entanglement. J Opt B: Quantum Semiclass Opt, 2001, 3: 223-227

42 Acin A, Andrianov A, Costa L, et al. Generalized Schmidt decomposition and classification of three-quantum-bit states. Phys Rev Lett, 2000, 85: 1560-1563
43 Horodecki M, Horodecki P, Horodecki R. Mixed-state entanglement and distillation: Is there a bound entanglement in nature? Phys Rev Lett, 1998, 80: 5239-5242

44 Peres A. Separability criterion for density matrices. Phys Rev Lett, 1996, 77: 1413-1415

45 Życzkowski K, Horodecki P. Volume of the set of separable states. Phys Rev A, 1998, 58: 883-892

46 Horodecki M, Horodecki P, Horodecki R. Separability of mixed states: necessary and sufficient conditions. Phys Lett A, 1996, 223: 1-8

47 Yu S X, Pan J W, Chen Z B, et al. Comprehensive test of entanglement for two-level systems via the indeterminacy relationship. Phys Rev Lett, 2003, 91: 217903

48 Cabello A. Proposal for revealing quantum nonlocality via local contextuality. Phys Rev Lett, 2010, 104: 220401

49 Amselem E, Rådmark M, Bourennane M, et al. State-independent quantum contextuality with single photons. Phys Rev Lett, 2009, 103: 160405

Open Access This article is distributed under the terms of the Creative Commons Attribution License which permits any use, distribution, and reproduction in any medium, provided the original author(s) and source are credited. 